\title{
LEFT JORDAN DERIVATIONS ON BANACH ALGEBRAS AND RELATED MAPPINGS
}

\author{
Yong-SoO JunG* and KyoO-Hong PARK
}

\begin{abstract}
In this note, we obtain range inclusion results for left Jordan derivations on Banach algebras: (i) Let $\delta$ be a spectrally bounded left Jordan derivation on a Banach algebra $A$. Then $\delta$ maps $A$ into its Jacobson radical. (ii) Let $\delta$ be a left Jordan derivation on a unital Banach algebra $A$ with the condition $\sup \left\{r\left(c^{-1} \delta(c)\right): c \in A\right.$ invertible $\}<\infty$. Then $\delta$ maps $A$ into its Jacobson radical.

Moreover, we give an exact answer to the conjecture raised by Ashraf and Ali in [2, p. 260]: every generalized left Jordan derivation on 2-torsion free semiprime rings is a generalized left derivation.
\end{abstract}

\section{Introduction}

Throughout this note, $R$ will represent an associative ring with center $Z(R)$ and we will write $[a, b]$ for the commutator $a b-b a$. The Jacobson radical of $R$ which is the intersection of all primitive ideals of $R$ will be denoted by $\operatorname{rad}(R)$.

Recall that $R$ is semiprime (resp. prime) if $a R a=0$ implies $a=0$ (resp. $a R b=0$ implies $a=0$ or $b=0$ ) and that $R$ is semisimple if $\operatorname{rad}(R)=\{0\}$. An additive mapping $\delta: R \rightarrow R$ is called a derivation (resp. Jordan derivation) if $\delta(a b)=a \delta(b)+\delta(a) b$ for all $a, b \in R\left(\right.$ resp. $\delta\left(a^{2}\right)=a \delta(a)+\delta(a) a$ for all $\left.a \in A\right)$. Obviously, every derivation is a Jordan derivation. The converse, in general, is not true. Brešar [4] proved that every Jordan derivation on a 2-torsion free semiprime ring is a derivation.

An additive mapping $d: R \rightarrow R$ is said to be a left Jordan derivation or Jordan left derivation (resp. left derivation) if $d\left(a^{2}\right)=2 a d(a)$ for all $a \in R$ (resp. $d(a b)=a d(b)+b d(a)$ for all $a, b \in R)$. Brešar, Vukman ([7], [17]), Deng [8] and Ashraf et al. [1] studied left Jordan derivations and left derivations on prime rings and semiprime rings, which are in a close connection with so-called commuting mappings.

Received November 9, 2008.

2000 Mathematics Subject Classification. 46H99, 47B47, 16N60.

Key words and phrases. (generalized) left Jordan derivation, (generalized) left derivation, derivation, spectral boundedness, Jacobson radica.

* This work was supported by the Korea Research Foundation Grant funded by the Korean Government(KRF-2008-313-C00045). 
Now let us introduce some principal results concerning derivations and related mappings in Banach algebra theory. The non-commutative Singer-Wermer conjecture states that if $\delta$ is a linear derivation on a Banach algebra $A$ such that $[\delta(a), a] \in \operatorname{rad}(A)$ for all $a \in A$, then $\delta(A) \subseteq \operatorname{rad}(A)$. This is equivalent to the fact that all primitive ideals of $A$ are invariant under $\delta$ [9]. There is some evidence for the validity of the conjecture [16]. It is known to be true if $\delta$ is continuous ([7], [10]) or if $A$ is commutative [15], while the classical Singer-Wermer theorem [14] is affirmative if both hypotheses are satisfied. Also, as one of noncommutative versions of the Singer-Wermer theorem (for example, [9]), Brešar and Vukman [7] proved that every continuous linear left derivation on a Banach algebra $A$ maps $A$ into $\operatorname{rad}(A)$. And they raised the problem whether the above conclusion holds for any continuous linear left Jordan derivation [7]. In case $A$ is commutative, the problem is equivalent to the classical Singer-Wermer theorem.

In Section 2, we improve the results in [13] as non-commutative versions of the Singer-Wermer theorem. Moreover, in Section 3, we give an exact answer to a conjecture raised by Ashraf and Ali [2] and investigate the spectral boundedness of generalized left derivations.

\section{Range inclusion results for left Jordan derivations}

Definition 2.1. Let $A$ and $B$ be Banach algebras. A linear mapping $T: A \rightarrow$ $B$ is called spectrally bounded if there is $M \geq 0$ such that $r(T(a)) \leq M r(a)$ for all $a \in A$. If $r(T(a))=r(a)$ for all $a \in A$, we say that $T$ is a spectral isometry. If $r(a)=0$, then $a$ is called quasinilpotent. (Herein, $r(a)=\lim _{n \rightarrow \infty}\left\|a^{n}\right\|^{\frac{1}{n}}$ denotes the spectral radius of the element $a$ ).

Remark 2.2. Brešar and Mathieu [6] showed that if $\delta$ is a linear derivation on a unital Banach algebra $A$, then the three conditions ' $\delta$ is spectrally bounded', $' \sup \left\{r\left(c^{-1} \delta(c)\right) \mid c \in A\right.$ invertible $\}<\infty$ ' and ' $\delta(A) \subseteq \operatorname{rad}(A)$ ' are equivalent each other.

We proved the following results concerning left Jordan derivations in [13] motivated by the Brešar and Mathieu's results in Remark 2.2:

(i) Every spectrally bounded left Jordan derivation $\delta$ on a Banach algebra $A$ such that $[\delta(a), a] \in \operatorname{rad}(A)$ for all $a \in A$, maps $A$ into $\operatorname{rad}(A)$.

(ii) Every linear left Jordan derivation $\delta$ on a unital Banach algebra $A$ with the condition $\sup \left\{r\left(c^{-1} \delta(c)\right): c \in A\right.$ invertible $\}<\infty$ such that $[\delta(a), a] \in \operatorname{rad}(A)$ for all $a \in A, \operatorname{maps} A$ into $\operatorname{rad}(A)$.

Here we obtain our main results without the condition $[\delta(a), a] \in \operatorname{rad}(A)$ for all $a \in A$.

Theorem 2.3. Let $A$ be a Banach algebra. If $\delta$ is a spectrally bounded left Jordan derivation on $A$, then we have $\delta(A) \subseteq \operatorname{rad}(A)$. 
Proof. Let $r(\delta(a)) \leq M r(a)$ for some $M \geq 0$ and all $a \in A$. Since the canonical epimorphism $\pi: A \rightarrow A / \operatorname{rad}(A)$ is a spectral isometry, it follows from [7, Proposition $1.1\left(1^{\circ}\right)$ ] that

$$
\begin{aligned}
r(2 a \delta(b)) & =r(\delta(a b+b a)-2 b \delta(a)) \\
& =r(\pi(\delta(a b+b a)-2 b \delta(a))) \\
& =r(\pi(\delta(a b+b a)-\pi(2 b \delta(a))) \\
& =r(\pi(\delta(a b+b a))) \\
& =r(\delta(a b+b a)) \\
& \leq M r(a b+b a)=0, a \in A, b \in \operatorname{rad}(A) .
\end{aligned}
$$

Therefore we obtain that $r(a \delta(b))=0$ for all $a \in A$ and $b \in \operatorname{rad}(A)$ and so Proposition 1 in [3, p. 126] tells us that $\delta(\operatorname{rad}(A)) \subseteq \operatorname{rad}(A)$. Then we can define a linear left Jordan derivation $\bar{\delta}$ on the semisimple Banach algebra $A / \operatorname{rad}(A)$ by $\bar{\delta}(a+\operatorname{rad}(A))=\delta(a)+\operatorname{rad}(A)$ for all $a \in A$. Hence, from [19, Theorem 4], we conclude that $\bar{\delta}=0$, i.e., $\delta(A) \subseteq \operatorname{rad}(A)$. This completes the proof of the theorem.

Theorem 2.4. Let $A$ be a unital Banach algebra. If $\delta$ is a linear left Jordan derivation on $A$ with the condition $\sup \left\{r\left(c^{-1} \delta(c)\right): c \in A\right.$ invertible $\}<\infty$, then we have $\delta(A) \subseteq \operatorname{rad}(A)$.

Proof. Let $\pi: A \rightarrow A / \operatorname{rad}(A)$ be the canonical epimorphism. Let

$$
s=\sup \left\{r\left(c^{-1} d(c)\right): c \in A \text { invertible }\right\}<\infty .
$$

We claim that $\delta(\operatorname{rad}(A)) \subseteq \operatorname{rad}(A)$. Given $c \in \operatorname{rad}(A)$, we have $(1+c)^{-1}=$ $1-c(1+c)^{-1} \in 1+\operatorname{rad}(A)$ and hence

$$
\begin{aligned}
r\left((1+c)^{-1} d(1+c)\right) & =r\left(\left(1-c(1+c)^{-1}\right) \delta(c)\right) \\
& =r\left(\delta(c)-c(1+c)^{-1} \delta(c)\right) \\
& =r\left(\pi\left(\delta(c)-c(1+c)^{-1} \delta(c)\right)\right) \\
& =r\left(\pi(\delta(c))-\pi\left(c(1+c)^{-1} \delta(c)\right)\right) \\
& =r(\pi(\delta(c))) \\
& =r(\delta(c)) .
\end{aligned}
$$

By the assumption, it follows that $r(d(c)) \leq s<\infty$ for all $c \in \operatorname{rad}(A)$, hence $r(d(c))=0$ for all $c \in \operatorname{rad}(A)$. It follows from [7, Proposition $\left.1.1\left(1^{\circ}\right)\right]$ that

$$
\begin{aligned}
r(2 a \delta(b)) & =r(\delta(a b+b a)-2 b \delta(a)) \\
& =r(\pi(\delta(a b+b a)-2 b \delta(a))) \\
& =r(\pi(\delta(a b+b a)-\pi(2 b \delta(a))) \\
& =r(\pi(\delta(a b+b a))) \\
& =r(\delta(a b+b a))=0, a \in A, b \in \operatorname{rad}(A) .
\end{aligned}
$$


Therefore we see that $r(a \delta(b))=0$ for all $a \in A$ and $b \in \operatorname{rad}(A)$, and so $\delta(\operatorname{rad}(A)) \subseteq \operatorname{rad}(A)$, as claimed. The remainder follows the same fashion as in the proof of Theorem 2.3. Hence we obtain $\delta(A) \subseteq \operatorname{rad}(A)$. This completes the proof.

\section{Spectrally boundedness of generalized left derivations and generalized left Jordan derivations on semiprime rings}

An additive mapping $f: R \rightarrow R$ is called a generalized derivation (resp. generalized Jordan derivation) if there exists a derivation $\delta: R \rightarrow R$ (resp. a Jordan derivation $\delta: R \rightarrow R$ ) such that $f(a b)=a f(b)+\delta(a) b$ holds for all $a, b \in R$ (resp. $f\left(a^{2}\right)=a f(a)+\delta(a) a$ holds for all $a \in R$ ). The concept of generalized derivation has been introduced by Brešar [5]. Jing and $\mathrm{Lu}$ [11] proved that every generalized Jordan derivation on 2-torsion free prime ring is a generalized derivation. In case when $R$ is semiprime, they conjectured that the result above may be still true, and Vukman [18] proved the conjecture.

An additive mapping $g: R \rightarrow R$ is called a generalized left derivation (resp. generalized left Jordan derivation) if there exists a left derivation $d: R \rightarrow R$ (resp. a left Jordan derivation $d: R \rightarrow R$ ) such that $g(a b)=a g(b)+b d(a)$ holds for all $a, b \in R$ (resp. $g\left(a^{2}\right)=a g(a)+a d(a)$ holds for all $a \in R$ ).

Brešar and Mathieu [6, Theorem 2.8] obtained a necessary and sufficient condition for a generalized derivation to be spectrally bounded on a unital Banach algebra:

Let $f=\tau_{t}+\delta$ be a generalized derivation with $t=f(1)$ associated with a derivation $\delta$ on a unital Banach algebra $A$, where $\tau_{t}$ is a left multiplication by t. The following conditions are equivalent.

(i) $f$ is spectrally bounded.

(ii) Both $\tau_{t}$ and $\delta$ are spectrally bounded.

Let $R$ be a ring. It is easy to prove that $g: R \rightarrow R$ is a generalized left derivation if and only if $g$ is of the form $g=\lambda+d$, where $\lambda: R \rightarrow R$ is a right centralizer and $d: R \rightarrow R$ is a left derivation. If $R$ contains a unit element, then it is easy to see that $g$ is a of the form $g=\mu_{k}+d$, where $\mu_{k}$ is a right multiplication by $k=\lambda(1)$. We here apply the Brešar and Mathieu's result above to arbitrary spectrally bounded generalized left derivations.

Theorem 3.1. Let $g=\mu_{k}+d$ be a generalized left derivation with $k=\lambda(1)$ on a unital Banach algebra $A$. The following conditions are equivalent.

(i) $g$ is spectrally bounded.

(ii) Both $\mu_{k}$ and d are spectrally bounded.

Proof. Let $\pi: A \rightarrow A / \operatorname{rad}(A)$ be the canonical epimorphism. Suppose that both $\mu_{k}$ and $d$ are spectrally bounded. By [12, Theorem 3.12], $d(A) \subseteq \operatorname{rad}(A)$ 
whence

$$
\begin{aligned}
r(g(a)) & =r\left(\mu_{k}(a)+d(a)\right) \\
& =r\left(\pi\left(\mu_{k}(a)+d(a)\right)\right) \\
& =r(\pi(a k+\pi(d(a))) \\
& =r(\pi(a k)) \\
& =r(a k) \leq \operatorname{Mr}(a)
\end{aligned}
$$

for some $M \geq 0$ and all $a \in A$. Hence $g$ is spectrally bounded.

Conversely, suppose that $g$ is spectrally bounded. It suffices to show that $d$ is spectrally bounded. For then, since we know that $d(A) \subseteq \operatorname{rad}(A)$ by $[12$, Theorem 3.12] and that $r(a k)=r(g(a))$ for all $a \in A$ as the above relation, it follows that $\mu_{k}$ is spectrally bounded with the same constant as $g$.

From the relation

$$
\begin{aligned}
r(a d(b)) & =r(d(a b)-b d(a)) \\
& =r(g(a b)-a b k-b d(a)) \\
& =r(\pi(g(a b)-a b k-b d(a))) \\
& =r(\pi(g(a b))-\pi(a b k)-\pi(b d(a))) \\
& =r(\pi(g(a b))) \\
& =r(g(a b)) \leq M r(a b)=0
\end{aligned}
$$

for some $M \geq 0$ and for all $a \in A$ and $b \in \operatorname{rad}(A)$, we arrive at

$$
r(a d(b))=0
$$

for all $a \in A$ and $b \in \operatorname{rad}(A)$ which implies that

$$
d(\operatorname{rad}(A)) \subseteq \operatorname{rad}(A) .
$$

Since every left derivation on semisimple Banach algebras is zero by [12, Corollary 3.7], the induced derivation on the semisimple Banach algebra $A / \operatorname{rad}(A)$ yields $d(A) \subseteq \operatorname{rad}(A)$. Therefore, $d$ is spectrally bounded by [12, Theorem 3.12].

An additive mapping $\lambda: R \rightarrow R$ is called a left (resp. right) centralizer if $\lambda(a b)=\lambda(a) b($ resp. $\lambda(a b)=a \lambda(b))$ holds for all $a, b \in R$. An additive mapping $\lambda: R \rightarrow R$ is called left (resp. right) Jordan centralizer if $\lambda\left(a^{2}\right)=\lambda(a) a$ (resp. $\left.\lambda\left(a^{2}\right)=a \lambda(a)\right)$ holds for all $a \in R$.

Obviously, every left (resp. right) centralizer is a left (resp. right) Jordan centralizer. Zalar has proved the following fact.

Lemma 3.2 ([20, Proposition 1.4]). Let $R$ be a 2-torsion free semiprime ring. If $\lambda: R \rightarrow R$ is a left (resp. right) Jordan centralizer, then $\lambda$ is a left (resp. right) centralizer. 
Recently, Ashraf and Ali [2] proved that every generalized left Jordan derivation on prime rings is a generalized left derivation. In [2], they also conjectured that every generalized left Jordan derivation on semiprime rings may be a generalized left derivation. Finally, we give an exact answer to this conjecture:

Theorem 3.3. Let $R$ be a 2-torsion free semiprime ring. If $g: R \rightarrow R$ is a generalized left Jordan derivation, then $g$ is a generalized derivation.

Proof. Suppose that there exists $d: R \rightarrow R$ is a left Jordan derivation such that

$$
g\left(a^{2}\right)=a g(a)+a d(a)
$$

is fulfilled for all $a \in R$. Let us denote $g-d$ by $\lambda$. Using the relation above, we get

$$
\begin{aligned}
\lambda\left(a^{2}\right) & =g\left(a^{2}\right)-d\left(a^{2}\right) \\
& =a g(a)+a d(a)-2 a d(a) \\
& =a(g(a)-d(a)) \\
& =a \lambda(a)
\end{aligned}
$$

for all $a \in R$. We have therefore $\lambda\left(a^{2}\right)=a \lambda(a)$ for all $a \in R$. In other words, $\lambda$ is a right Jordan centralizer of $R$. Since $R$ is a 2-torsion free semiprime ring, it follows from [19, Theorem 2] and Lemma 3.2 that $d: R \rightarrow R$ is a derivation such that $d(R) \subseteq Z(R)$ and $\lambda$ is a right centralizer of $R$, respectively. Since we know that $g=\lambda+d$, we see that the equality $g(a b)=a g(b)+d(a) b$ holds for all $a, b \in R$. That is, we conclude that $g$ is a generalized derivation. The proof of the theorem is complete.

\section{References}

[1] M. Ashraf, On Jordan left derivations of Lie ideals in prime rings, Southeast Asian Bull. Math. 25 (2001), no. 3, 379-382.

[2] M. Ashraf and S. Ali, On generalized Jordan left derivations in rings, Bull. Korean Math. Soc. 45 (2008), no. 2, 253-261.

[3] F. F. Bonsall and J. Duncan, Complete Normed Algebras, Ergebnisse der Mathematik und ihrer Grenzgebiete, Band 80. Springer-Verlag, New York-Heidelberg, 1973.

[4] M. Brešar, Jordan derivations on semiprime rings, Proc. Amer. Math. Soc. 104 (1988), no. 4, 1003-1006.

[5] _ On the distance of the composition of two derivations to the generalized derivations, Glasgow Math. J. 33 (1991), no. 1, 89-93.

[6] M. Brešar and M. Mathieu, Derivations mapping into the radical. III, J. Funct. Anal. 133 (1995), no. 1, 21-29.

[7] M. Brešar and J. Vukman, On left derivations and related mappings, Proc. Amer. Math. Soc. 110 (1990), no. 1, 7-16.

[8] Q. Deng, On Jordan left derivations, Math. J. Okayama Univ. 34 (1992), 145-147

[9] M. Mathieu, Where to find the image of a derivation, Functional analysis and operator theory (Warsaw, 1992), 237-249, Banach Center Publ., 30, Polish Acad. Sci., Warsaw, 1994.

[10] M. Mathieu and G. J. Murphy, Derivations mapping into the radical, Arch. Math. (Basel) 57 (1991), no. 5, 469-474. 
[11] W. Jing and S. Lu, Generalized Jordan derivations on prime rings and standard operator algebras, Taiwanese J. Math. 7 (2003), no. 4, 605-613.

[12] Y.-S. Jung, On left derivations and derivations of Banach algebras, Bull. Korean Math. Soc. 35 (1998), no. 4, 659-667.

[13] _ Some results on Jordan left derivations in Banach algebras, Commun. Korean Math. Soc. 14 (1999), no. 3, 513-519.

[14] I. M. Singer and J. Wermer, Derivations on commutative normed algebras, Math. Ann. 129 (1955), 260-264.

[15] M. P. Thomas, The image of a derivation is contained in the radical, Ann. of Math. (2) 128 (1988), no. 3, 435-460.

[16] - Primitive ideals and derivations on noncommutative Banach algebras, Pacific J. Math. 159 (1993), no. 1, 139-152.

[17] J. Vukman, Jordan left derivations on semiprime rings, Math. J. Okayama Univ. 39 (1997), 1-6.

[18] A note on generalized derivations of semiprime rings, Taiwanese J. Math. 11 (2007), no. 2, 367-370.

[19] On left Jordan derivations of rings and Banach algebras, Aequationes Math. 75 (2008), no. 3, 260-266.

[20] B. Zalar, On centralizers of semiprime rings, Comment. Math. Univ. Carolin. 32 (1991), no. 4, 609-614.

YONG-SOO JUNG

Department of Mathematics

Sun MOON UNIVERSITY

Chungnam 336-708, Korea

E-mail address: ysjung@sunmoon.ac.kr

KYOO-HONG PARK

Department of Mathematics Education

SEOWON UNIVERSITY

Chungbuk 361-742, KoreA

E-mail address: parkkh@seowon.ac.kr 